\title{
Operating Room Efficiency for General Anesthesia Cases in the Department of Ophthalmology in a Public Tertiary Hospital
}

\author{
Maria Isabel N. Umali, MD and Teresita R. Castillo, MD, MHPEd \\ Department of Ophthalmology and Visual Sciences, Philippine General Hospital, University of the Philippines Manila
}

\begin{abstract}
Objective. To determine operating room efficiency for elective ophthalmologic surgeries requiring general anesthesia in a public tertiary institution based on standard efficiency parameters.

Methods. Prospective observational cross-sectional study of randomly selected elective cases requiring general anesthesia from April 2019 to June 2019 in the Department of Ophthalmology of the Philippine General Hospital. A single third-party observer recorded operating room milestones from which efficiency parameters were determined and compared with local and international guidelines and efficiency benchmarks.

Results. A total of fifty cases from the Retina, Plastic, Orbit, Glaucoma, and Motility services were observed. None started on the specified start time of 6:30 a.m., with surgeries starting an average of $52 \pm 11.90$ minutes after. Across subspecialties, median surgical preparation time was statistically significant $\left(\chi^{2}: 12.01, p: 0.02\right)$, with the Retina and Orbit services having the most extended duration. Across age groups, pediatric cases had lower mean anesthesia preparation times (t: 2.15 , df: 48, p: 0.04) and median trans-out lag times ( $\left.\chi^{2}: 4.56, p: 0.03\right)$ than adults. Overall, more than $60 \%$ of cases reached targets for induction and surgical lag time. Turnaround for adult and pediatric patients was $75 \pm 22.77$ minutes and $71 \pm 14.91$ minutes, respectively. Benchmarking analysis showed that the first case on time, entry lag, and exit lag were below the $50^{\text {th }}$ percentile while the room turnover time was above the $95^{\text {th }}$ percentile.
\end{abstract}

Conclusion. Ensuring efficiency requires a multidisciplinary team approach. This research can guide administrators in determining interventions to increase operating room efficiency.

Key Words: operating room, efficiency, ophthalmology

\section{INTRODUCTION}

The Operating Room (OR) is a vital asset in any tertiary hospital, as it contributes to more than two-thirds of total hospital revenue. ${ }^{1}$ At the same time, $40 \%$ of the hospital's expenses, including both workforce and operating costs, come from the operating room. ${ }^{2}$ Thus, the different processes happening in the OR must be efficient to boost profitability and minimize cost while retaining the quality of surgical care.

Efficiency in the operating room can be determined

Presented at the Philippine Academy of Ophthalmology Annual Convention, December 8, 2019, SMX Mall of Asia, Manila.

Corresponding author: Maria Isabel N. Umali, MD

Department of Ophthalmology and Visual Sciences Philippine General Hospital

University of the Philippines Manila

Taft Avenue, Manila 1000, Philippines

Email: mnumali1@up.edu.ph through variable methods and measured by different parameters. The times of the operating room milestones can be taken through direct observation as the processes are taking place or through a previous recording, such as in closed-circuit television (CCTV). The times taken from these milestones can then be used to compute the different efficiency parameters. Likewise, factors such as surgical 
scheduling accuracy, punctuality, procedure time variation, and inter-operative delays, among others, influence how time is used in the operating room. ${ }^{3}$

The Philippine General Hospital Department of Ophthalmology houses its operating room complex on the fourth floor. The complex comprises six operating rooms, five used for major operations and one for minor operations, and its post-anesthesia care unit (PACU) with four beds. The operating room caters to both pay and charity cases. Over the last year, there has been a total of over five thousand surgeries, with total earnings reaching Php 18 million.

At present, there are no available local data on operating room efficiency for ophthalmologic surgeries. This study aims to measure operating room efficiency through different parameters and compare these with local and international published guidelines. Results can then serve as a baseline for future research and guide administrators in determining improvements in efficiency and quality health care.

\section{METHODS}

This is a prospective observational study done at the Operating Room (OR) Complex of the Department of
Ophthalmology of the Philippine General Hospital from April to June 2019. Elective surgeries requiring general anesthesia were included, while private surgeries performed by consultants and emergency cases were excluded. Surgeries done with residents of other departments were also not included.

\section{Data Collection}

Randomly selected rooms with scheduled elective cases requiring general anesthesia were observed by a single third-party observer who collected data using a standard time-taking device. The observer is an ophthalmologistin-training who was not employed at the hospital where the study was conducted. Likewise, the hospital staff knew of an ongoing efficiency study but were not aware of the exact date of the start of data collection nor the specific room being observed.

Demographic data collected include the date, age, gender, surgeon, anesthesiologist, subspecialty service, and surgical procedure. Patient and support staff (i.e., nurses, utility workers) identifiers were not collected while doctor identifiers were anonymized and codified. The different operating room milestones were noted, and the efficiency metrics were calculated (Tables 1 and 2).

Table 1. Operating room milestones observed in the study, with their abbreviations and definitions. Modified from the Glossary of the Association of Anesthesia Clinical Directors ${ }^{5}$ and Lapitan, et al. ${ }^{6}$

\begin{tabular}{lcl}
\multicolumn{1}{c}{ Milestone } & Abbreviation & Definition \\
Patient in room & PIR & Time patient is wheeled inside the room \\
Anesthesia prep start & APS & Time anesthesiologist starts placing monitoring devices on patient \\
Anesthesia start & AS & Time anesthesiologist injects intravenous anesthetic \\
Anesthesia ready & AR & Time anesthesiologist hands over the patient to surgeons \\
Surgical preparation start & SPS & Time surgeons start positioning and marking the patient \\
Surgical preparation end & SPE & Time betadine wash is finished \\
Procedure start time & PST & Time of placement of lid retractors or first incision for gross surgeries \\
Procedure finish time & PFT & Time lid retractors are removed or final dressing placed for gross surgeries \\
Patient ready for trans-out & PRTO & Time anesthesiologist announces that the patient can be transferred to PACU \\
Patient out of operating room & POR & Time patient is wheeled out of the operating room \\
\hline
\end{tabular}

Table 2. Efficiency indicators with corresponding abbreviations, mathematical derivations, definitions, and set standard time (in minutes). Modified from the Glossary of the Association of Anesthesia Clinical Directors ${ }^{5}$ and Lapitan, et al. ${ }^{6}$

\begin{tabular}{|c|c|c|c|}
\hline $\begin{array}{l}\text { Efficiency Indicator } \\
\text { (abbreviation) }\end{array}$ & Data Source & Definition & $\begin{array}{c}\text { Target } \\
\text { (minutes) }\end{array}$ \\
\hline Anesthesia lag time (ALT) & $A L T=A P S-P I R$ & Time from patient entry into OR to start of anesthesia preparation & 0 \\
\hline $\begin{array}{l}\text { Anesthesia Preparation } \\
\text { Time (APT) }\end{array}$ & APT $=$ AS- APS & $\begin{array}{l}\text { Time from when anesthesiologist arrives and receives the patient at the OR } \\
\text { until the start of anesthesia induction (IV injection of anesthetic) }\end{array}$ & 5 \\
\hline Anesthesia induction (AI) & $A I=A R-A S$ & $\begin{array}{l}\text { Time spent on administration of anesthesia, from start of injection of } \\
\text { IV anesthetic to time patient is handed over for surgical prep }\end{array}$ & 15 \\
\hline Surgical prep time (SPT) & $\mathrm{SPT}=\mathrm{SPE}-\mathrm{SPS}$ & $\begin{array}{l}\text { Time spent on prepping and positioning of the patient for surgery; time from } \\
\text { surgical prep start time to end time }\end{array}$ & 10 \\
\hline Surgical lag time (SLT) & SLT $=$ PST - SPE & Time from end of surgical prepping to start of the operation & 5 \\
\hline Operative time (ORT) & ORT = PFT - PST & Total time of the surgery, time from procedure start time to procedure end time & $\mathrm{n} / \mathrm{a}$ \\
\hline Wake up time (WT) & WT $=$ PRTO - PFT & Time from end of surgery to complete emergence from anesthesia & 15 \\
\hline Trans-out lag time (TLT) & TLT = POR - PRTO & The from complete emergence from anesthesia to patient out of operating room & 0 \\
\hline Turnover time (TOT) & TOT = PIR2 - POR & Time from exit of patient until entry of the next patient to the operating room & 30 \\
\hline
\end{tabular}


The percentage of the first case on time and the number of minutes from the scheduled start time the first cases started were also determined. From all these data, the mean and median times for the measured OR efficiency parameters were calculated. Descriptive statistics were used for categorical data variables. Likewise, causes of delays were also noted by the observer.

\section{Data and Statistical Analysis}

Data was manually entered in a Microsoft Excel file and analyzed statistically using Stata 13 . The analysis considered the dataset in its entirety across ophthalmologic services and by their case sequence. A series of median tests and chi-square tests of association were performed to determine differences in the efficiency parameters across the groups, across services, and age groups. Independent t-tests were used to determine differences between age groups, but a one-way analysis of variance was used for the types of ophthalmologic services. Fisher-Hayter standardized multiple comparisons procedure was performed to determine between-group differences among those items where the ANOVA was significantly different.

Multiple linear regression analysis was performed, which did not involve variable selection methods since the current study aimed to explore clinical-process relevant associations. The assumptions of linearity, normality, and independence were assessed before the performance of the said analytic method. The level of significance for all sets of analyses was set at a p-value of less than 0.05 using two-tailed comparisons.

\section{Ethical Considerations}

The study commenced upon approval of the University of the Philippines, Manila Ethics Review Board and is a recipient of the Philippine General Hospital Expanded Health Research Office (EHRO) research grant. There are no conflicts of interest. The study was conducted according to the provisions of the Declaration of Helsinki.

\section{RESULTS}

A total of 50 surgeries were observed for the specified period. Ophthalmologic subspecialties observed in this study included Retina, Plastic/ Lacrimal, Orbit, Glaucoma, and
Motility. Overall, there were more female patients $(n=28$, $56 \%)$ and adult cases $(n=32,64 \%)$ observed (Table 3$)$.

There was no case observed which started on the specified start time of 6:30 a.m. The number of minutes late from the expected start time of the procedure was significantly lower in the Motility service $(F(4,24), p<0.01)$. The difference in median surgical preparation time was statistically significant $\left(\chi^{2}: 12.01, \mathrm{p}: 0.02\right)$, with the Retina and Orbit services having the most prolonged duration for this parameter (13 minutes) (Table 4). In terms of percentage of cases reaching target times, the parameters of anesthesia lag time and surgical preparation time were significantly different across services $(\mathrm{p}=0.02)$, with Motility service achieving the anesthesia lag time target for all its patients. However, the anesthesia preparation time, induction time, surgical lag time, wakeup time, and trans-out lag time were not statistically significant. All services had $60 \%$ of their cases reaching the target for the parameters of surgical lag and wakeup times (Figure 1).

Comparing efficiency parameters according to age group showed that pediatric cases have lower mean anesthesia preparation times than adults (t: 2.15 , df: $48, \mathrm{p}: 0.04$ ). Likewise, the median trans-out lag times were significantly lower among pediatric patients ( $\chi^{2}: 4.56, \mathrm{p}: 0.03$ ) (Table 5). Overall, there was no significant difference between adult and pediatric patients in terms of percentage of cases reaching the target times, with greater than $60 \%$ of cases reaching targets for the parameters anesthesia lag time, induction time, surgical lag time, and wakeup time (Figure 2).

Segment analysis, which involved total turnaround time computation and multiple linear regression in predicting total turnaround time, was done for each age group (Figure 3). Turnaround for adult and pediatric patients was $75 \pm 22.77$ minutes and $71 \pm 14.91$ minutes, respectively. For adults, a significant regression equation was found ( $\mathrm{F}$ $(1,16)=5.30$, p: 0.04$)$, with a 0.20 coefficient of variation. The time from patient in until surgery start ("entry lag") was found to be a significant predictor for turnaround time for these patients $(95 \%$ CI $[0.58,1.52], \mathrm{p}<0.01)$. Likewise, a significant regression equation was also found among pediatric patients $(\mathrm{F}(1,8)=12.91, \mathrm{p}<0.01)$, with a 0.57 coefficient of variation. The duration from patient out to patient in ("turnover time"), and from patient in until surgery start ("entry lag") were found to be significant predictors for

Table 3. Demographic distribution of cases observed across the different subspecialties

\begin{tabular}{|c|c|c|c|c|c|c|}
\hline & $\begin{array}{c}\text { Overall } \\
\text { N (\%) }\end{array}$ & $\begin{array}{l}\text { Pedia } \\
\text { N (\%) }\end{array}$ & $\begin{array}{c}\text { Plastics } \\
\text { N (\%) }\end{array}$ & $\begin{array}{l}\text { Retina } \\
\text { N (\%) }\end{array}$ & $\begin{array}{c}\text { Glaucoma } \\
\mathbf{N}(\%)\end{array}$ & $\begin{array}{l}\text { Orbit } \\
N(\%)\end{array}$ \\
\hline \multicolumn{7}{|l|}{ Gender } \\
\hline Female & $28(56 \%)$ & 9 (32.14\%) & 7 (25\%) & 5 (17.86\%) & 3 (10.71\%) & 4 (14.29\%) \\
\hline Male & 22 (44\%) & 2 (9.09\%) & 5 (22.73\%) & 7 (31.82\%) & 5 (22.73\%) & 3 (13.64\%) \\
\hline \multicolumn{7}{|l|}{ Age Group } \\
\hline Adult & 32 (64\%) & 2 (6.25\%) & 8 (25\%) & 9 (28.13\%) & 7 (21.88\%) & 6 (18.75\%) \\
\hline Pediatric & $18(36 \%)$ & $9(50 \%)$ & $4(22.22 \%)$ & $3(16.67 \%)$ & $1(5.56 \%)$ & $1(5.56 \%)$ \\
\hline Total & 50 (100\%) & $11(22 \%)$ & $12(24 \%$ & $12(24 \%)$ & $8(16 \%)$ & $7(14 \%)$ \\
\hline
\end{tabular}




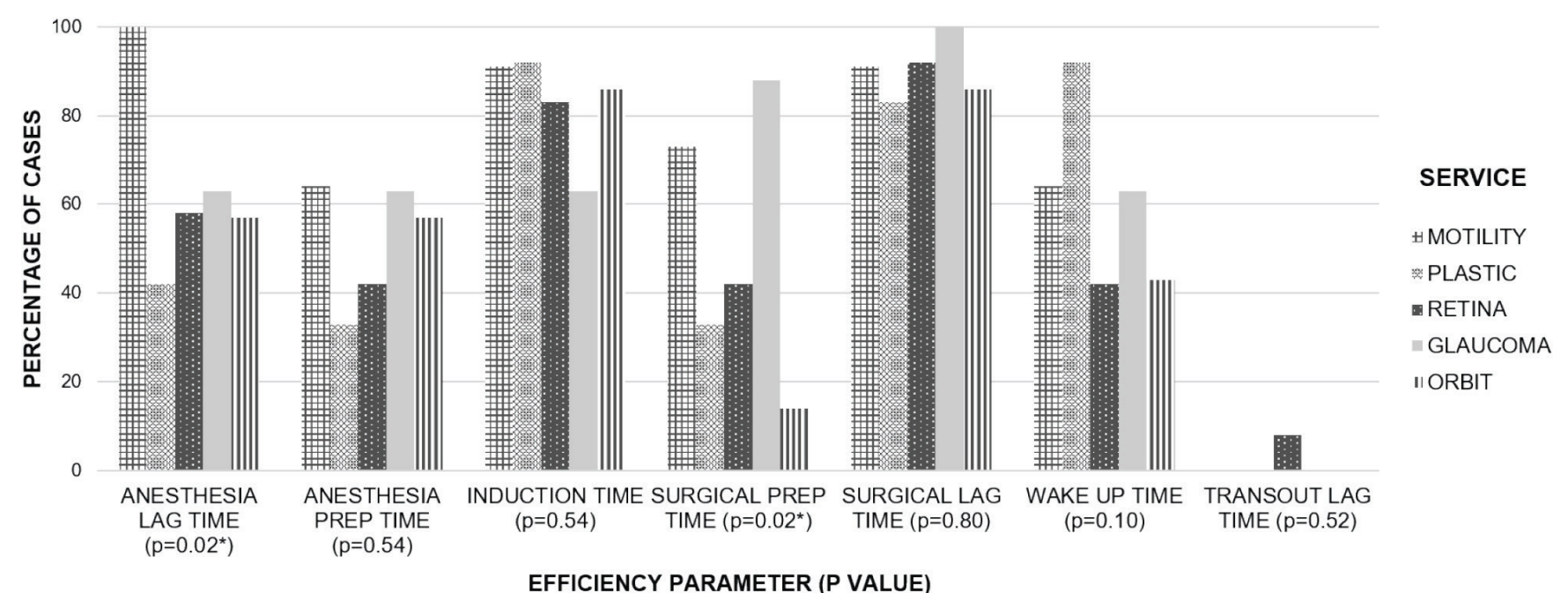

Figure 1. Percentage of cases reaching target time for the different efficiency parameters according to subspecialty service. ( ${ }^{*}$ statistically significant).

the said outcome in this age group (95\% CI [0.53, 2.42], $\mathrm{p}<0.01),(95 \% \mathrm{CI}[1.75,2.55], \mathrm{p}<0.01$, respectively).

Lastly, comparing results of this study with the OR Benchmarks Collaborative (ORBC), ${ }^{4}$ an automated benchmarking service from McKesson Enterprise Intelligence, showed that the department is below the $50^{\text {th }}$ percentile in terms of the key performance indicators of first case on time, entry lag and exit lag, but is above the $95^{\text {th }}$ percentile for room turnover time (Table 6).

\section{DISCUSSION}

\section{Current Department Practice}

The operating room complex opens at 6:00 a.m. The residents bring the patients scheduled for the first case to the OR at this time. The scheduled start time is 6:30 a.m., while the cut-off for elective cases using general anesthesia is 11:45 a.m., after which the OR caters to emergency cases. The anesthesiologists come from the PGH main

Table 4. Mean and median values for operating room efficiency parameters (in minutes) across all subspecialty services

\begin{tabular}{|c|c|c|c|c|c|c|c|}
\hline Parameter & Overall & Motility & Plastic & Retina & Glaucoma & Orbit & $p$-value \\
\hline Minutes Late \pm SD & $52 \pm 11.90$ & $38 \pm 12.05$ & $60 \pm 4.90$ & $60 \pm 8.72$ & $49 \pm 9.65$ & $51 \pm 9.87$ & $<0.01^{*}$ \\
\hline \multicolumn{8}{|c|}{ Anesthesia lag time (minutes) } \\
\hline Mean \pm SD & $8 \pm 9.20$ & $3 \pm 1.55$ & $12 \pm 13.18$ & $8 \pm 5.33$ & $5 \pm 1.64$ & $13 \pm 13.75$ & 0.06 \\
\hline Median & 5 & 2 & 10 & 5 & 5 & 5 & 0.06 \\
\hline \multicolumn{8}{|c|}{ Anesthesia prep time (minutes) } \\
\hline Mean \pm SD & $8 \pm 5.66$ & $5 \pm 1.29$ & $10 \pm 5.90$ & $9 \pm 7.98$ & $6 \pm 2.78$ & $8 \pm 5.40$ & 0.11 \\
\hline Median & 6 & 4 & 9 & 7 & 5 & 5 & 0.53 \\
\hline \multicolumn{8}{|l|}{ Induction time (minutes) } \\
\hline Mean \pm SD & $13 \pm 7.38$ & $11 \pm 4.41$ & $13 \pm 4.93$ & $13 \pm 11.16$ & $16 \pm 9.65$ & $14 \pm 3.91$ & 0.79 \\
\hline Median & 11 & 10 & 11 & 10 & 12 & 13 & 0.29 \\
\hline \multicolumn{8}{|c|}{ Surgical prep time (minutes) } \\
\hline Mean \pm SD & $13 \pm 6.65$ & $9 \pm 2.52$ & $13 \pm 4.11$ & $16 \pm 10.48$ & $10 \pm 7.23$ & $13 \pm 2.56$ & 0.14 \\
\hline Median & 11 & 9 & 12 & 13 & 8 & 13 & $0.02^{*}$ \\
\hline \multicolumn{8}{|l|}{ Surgical lag time (minutes) } \\
\hline Mean \pm SD & $3 \pm 1.81$ & $3 \pm 1.70$ & $3 \pm 2.00$ & $4 \pm 2.19$ & $3 \pm 7.23$ & $3 \pm 1.68$ & 0.58 \\
\hline Median & 3 & 3 & 2 & 4 & 2 & 3 & 0.11 \\
\hline \multicolumn{8}{|l|}{ Wake up time (minutes) } \\
\hline Mean \pm SD & $16 \pm 10.18$ & $19 \pm 16.63$ & $13 \pm 3.60$ & $19 \pm 10.72$ & $14 \pm 6.98$ & $17 \pm 5.97$ & 0.51 \\
\hline Median & 15 & 15 & 13 & 17 & 14 & 16 & 0.11 \\
\hline \multicolumn{8}{|c|}{ Trans-out lag time (minutes) } \\
\hline Mean \pm SD & $2 \pm 1.84$ & $2 \pm 0.83$ & $3 \pm 1.86$ & $2 \pm 1.23$ & $3 \pm 3.54$ & $2 \pm 1.07$ & 0.57 \\
\hline Median & 2 & 2 & 3 & 3 & 2 & 2 & 0.34 \\
\hline
\end{tabular}

Legend: *significant 
Table 5. Mean and median values for operating room efficiency parameters (in minutes) across age groups

\begin{tabular}{|c|c|c|c|}
\hline Parameters & Adult & Pediatric & p-value \\
\hline \multicolumn{4}{|c|}{ Anesthesia lag time (minutes) } \\
\hline Mean \pm SD & $9 \pm 10.64$ & $6 \pm 5.74$ & 0.35 \\
\hline Median & 5 & 5 & 0.55 \\
\hline \multicolumn{4}{|c|}{ Anesthesia prep time (minutes) } \\
\hline Mean \pm SD & $9 \pm 6.59$ & $5 \pm 2.23$ & $0.04^{*}$ \\
\hline Median & 6 & 5 & 0.77 \\
\hline \multicolumn{4}{|c|}{ Induction time (minutes) } \\
\hline Mean \pm SD & $14 \pm 8.52$ & $11 \pm 4.17$ & 0.12 \\
\hline Median & 12 & 10 & 0.50 \\
\hline \multicolumn{4}{|c|}{ Surgical prep time (minutes) } \\
\hline Mean \pm SD & $13 \pm 6.30$ & $12 \pm 7.39$ & 0.66 \\
\hline Median & 12 & 10 & 0.14 \\
\hline \multicolumn{4}{|c|}{ Surgical lag time (minutes) } \\
\hline Mean \pm SD & $3 \pm 1.91$ & $3 \pm 1.64$ & 0.82 \\
\hline Median & 3 & 3 & 0.43 \\
\hline \multicolumn{4}{|c|}{ Wake up time (minutes) } \\
\hline Mean \pm SD & $16 \pm 7.63$ & $17 \pm 13.86$ & 0.87 \\
\hline Median & 15 & 13 & 0.77 \\
\hline \multicolumn{4}{|c|}{ Trans-out lag time (minutes) } \\
\hline Mean \pm SD & $3 \pm 2.02$ & $2 \pm 1.41$ & 0.19 \\
\hline Median & 2 & 2 & $0.03^{*}$ \\
\hline
\end{tabular}

Legend: *significant building, connected to the department's facility by a bridge on the $4^{\text {th }}$ floor. They usually come from their duty at the main hospital's PACU or their department call room on the $3^{\text {rd }}$ floor. The primary surgeon is usually already in the operating room when the anesthesiologist arrives. The first-year resident is also usually advised by the primary surgeon to inform the anesthesiologist when to start induction in cases wherein the surgeon has not yet arrived.

However, in this study, it was not feasible for a single third-party observer to collect the anesthesiologist's exact arrival times and primary surgeons to the ophthalmology department OR complex.

At any given time, two anesthesiologists (usually one second-year and third-year resident each) are assigned to rotate in the department. They can perform simultaneous general anesthesia cases, provided that they are both adult patients. However, if a pediatric patient is scheduled, only the third-year anesthesiologist can perform that case. During the research period, an Anesthesiologist Medical Specialist (MS) was employed by the hospital, assigned to do ophthalmology cases once a week. The MS can perform both adult and pediatric patients. There are no set rules in the department regarding the start of induction. Some anesthesiologists demand the primary surgeon's presence, while others start preparation and induction as advised by the residents.

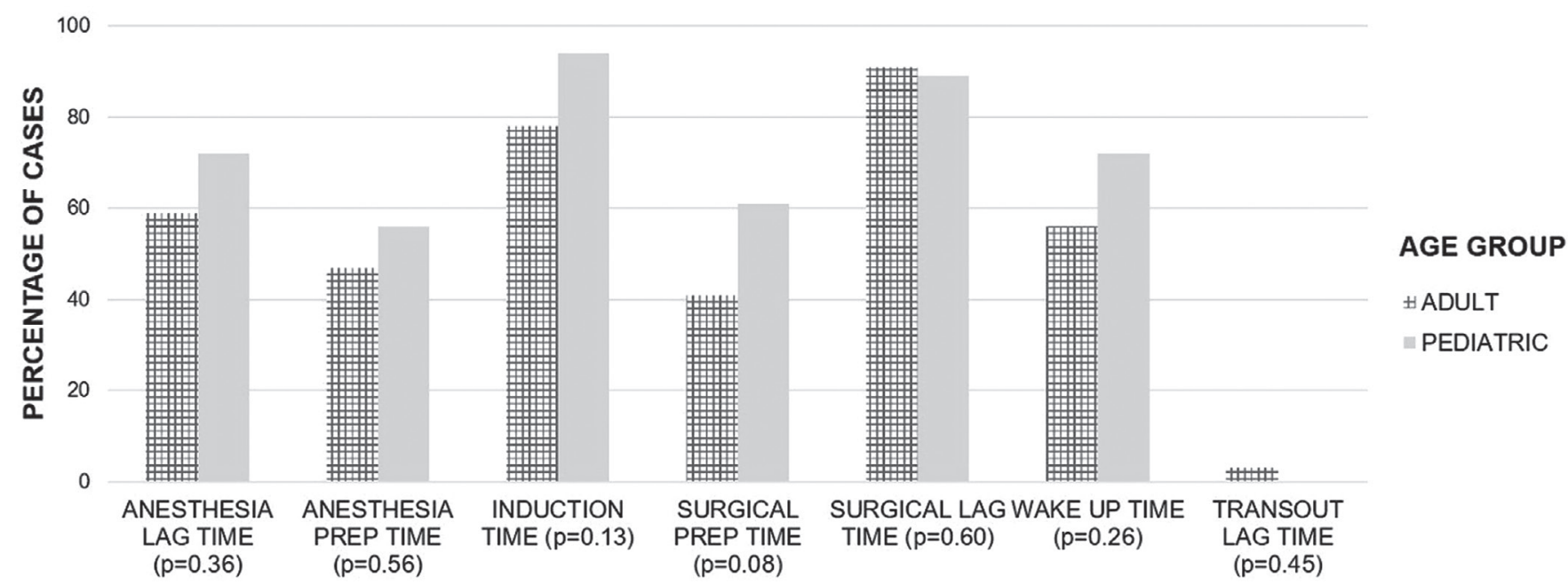

EFFICIENCY PARAMETER (P VALUE)

Figure 2. Percentage of cases reaching target time for the different efficiency parameters according to age group.

Table 6. Comparison of the key performance indicators computed in this study with that of OR Benchmarks Collaborative ${ }^{4}$

\begin{tabular}{lcccc} 
& Study data & \multicolumn{3}{c}{ Benchmark data $^{4}$} \\
\cline { 3 - 5 } Key performance indicator & (Adult subgroup) & Median & $\mathbf{9 0}^{\text {th }}$ percentile & $\mathbf{9 5}^{\text {th }}$ percentile \\
\hline First case on time / early (\%) & $0 \%$ & $64.3 \%$ & $88.3 \%$ & $91.4 \%$ \\
Entry lag (minutes \pm SD) & $39 \pm 16.60$ & 25.7 & 20.4 & 19.7 \\
Exit Lag (minutes \pm SD) & $17 \pm 9.59$ & 9.6 & 6.9 & 6.5 \\
Turnover time (minutes \pm SD) & $18 \pm 16.75$ & 28.5 & 22.7 & 21.4 \\
\hline
\end{tabular}




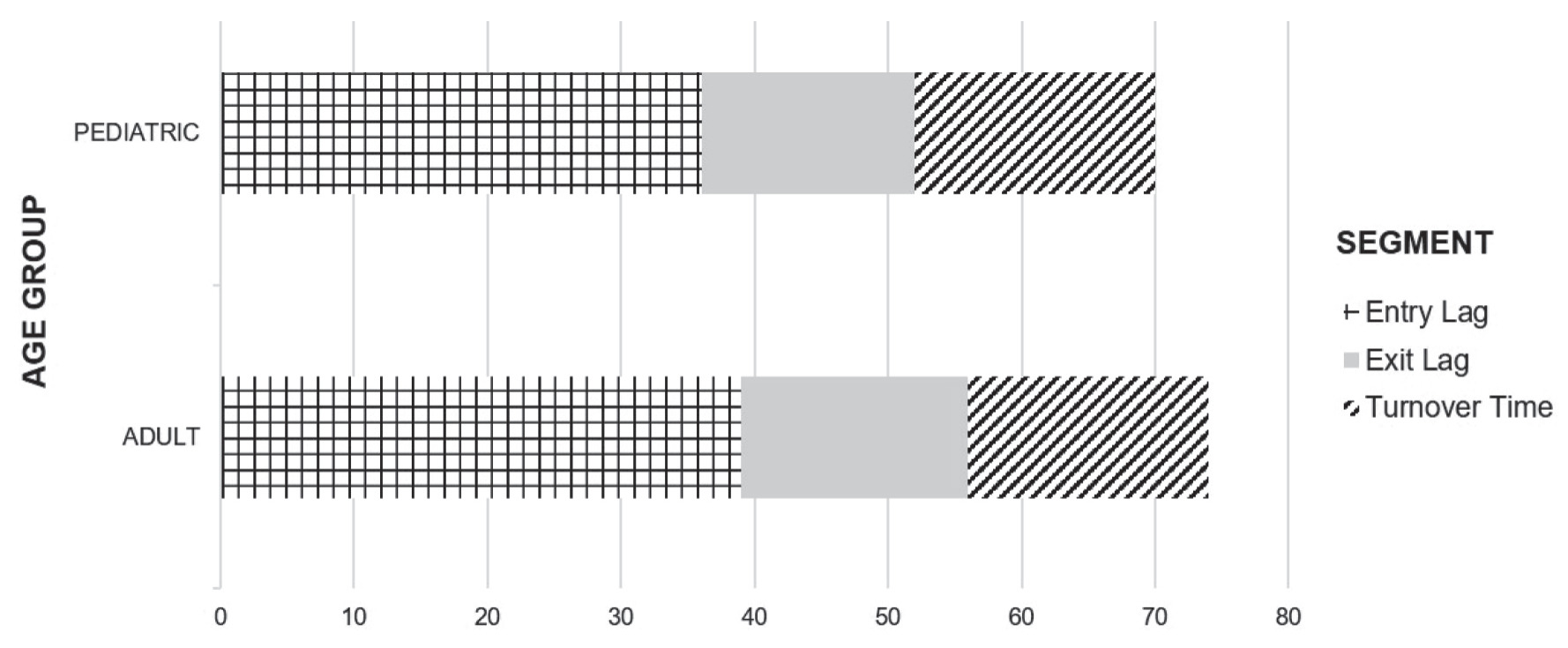

MINUTES

Figure 3. Turnaround time segment analysis according to age group. Turnaround time is composed of three segments, namely, in chronological order, exit lag, turnover time, and entry lag. Exit lag is the end of surgery until the exit of the patient; turnover time is the exit of the patient until the entry of the next patient; entry lag is the entry of the next patient until the start of surgery. Total turnaround time is the total of exit lag, turnover time, and entry lag segments and is from the exit of the first patient until the start of surgery of the next patient.

\section{Guidelines and Benchmarks Used}

As this study was the first for ophthalmologic surgeries in the country, no guidelines and targets for efficiency parameters have been set by the local specialty society. An earlier study done in the Department of Surgery of the same hospital, which included residents' cases, used the Association of Anesthesia Clinical Directors glossary ${ }^{5}$ and set their department standards. ${ }^{6}$ Since this study was similar to theirs; the same standard target times were used for this research. Likewise, there are also no available benchmarks for ophthalmologic surgeries in the country. For this purpose, The OR Benchmarks Collaborative ${ }^{4}$, a database with subscribers including acute care hospitals and ambulatory surgical centers in the United States, Canada, Saudi Arabia, Australia, and New Zealand, was chosen as the standard.

\section{Efficiency Parameters}

No case started at the specified start time in all fifty cases observed. The scheduled start "cutting" time is 6:30 a.m.; however, adding all the target times for the efficiency parameters gives a total of 45 minutes. The operating room complex opens at 6:00 a.m., providing 45 minutes for all the preparation needed for a general anesthesia case still did not yield any case which started on time. A reason may be because of a difference in the definition of the "start time." According to the target times set in the study done in the Department of Surgery ${ }^{6}, 6: 30$ a.m. is set as the time the patient arrives inside the room, while the procedure start time was 7:30 a.m. for first cases. Thus, there was a disconnect between the definitions of start time between the departments by one hour. Assuming that 7:30 a.m. is the start time that will be followed, 19 out of 25 first cases observed (76\%) will be starting on time. Regardless of the start time being followed, this finding highlights the need for a multidisciplinary approach and group goal setting to achieve efficiency. Furthermore, the Department of Ophthalmology has to either formally adopt the Department of Surgery standards. They are from the same hospital, employ the same anesthesiologists, or define their targets and make these standards known to all stakeholders involved.

The number of minutes late from the expected start time of the procedure was significantly lower in the Motility service than the other services. This may be because the Motility service's new setup is 9:00 a.m., cutting time after the department conference of the Anesthesia service, which ends at 8:30 a.m. This is also why the Motility service could reach the target time for anesthesia lag time. There are times that the anesthesiologists are not able to arrive at 6:00 a.m. at the operating room, especially if they are from duty and still have to endorse to the incoming duty team, thus delaying the start of the surgeries. Their duty ends at 6:15 a.m. then they still spend around 15-30 minutes endorsing to the next group, thus delaying their arrival to the OR complex by at least 30 minutes.

There is a wide range for anesthesia lag time for the different services since there are differences in the processes which affected the determination of the lag time. The anesthesia lag time is defined as the difference in time 
between anesthesia prep starts and when the patient is brought inside the room. The patient is usually wheeled in and prepped right away inside the operating room as soon as the OR complex opens at 6:00 a.m. Lately, it was practiced that the patient was only brought inside the operating room once the anesthesiologist arrives. If the first practice were done, the anesthesia lag time would increase to reflect the anesthesiologists' late arrival. However, since the second practice is done more commonly, the anesthesia lag time parameter will not reflect the anesthesiologist's possible late arrival. This study is limited because both surgeons and anesthesiologists' exact arrival time was not observed since a single observer could not note the movements of multiple doctors and patients simultaneously.

Commonly cited reasons for late arrival of the anesthesiologists include the distant location of their call room (in the other building), having to get supplies from the central pharmacy (also in the other building), and coming from a duty status and needing to endorse to the next duty team. With these factors in mind, the study clearly defines the procedure start time and other expected efficiency parameters to all stakeholders, especially the anesthesiologists, since they may have different practices in the other operating room complexes and hospital departments.

Anesthesia prep target time was not reached by the Plastic and Retina services. During the study period, the anesthesiologist assigned to these services was a Medical Specialist hired by the hospital. It was observed that there was difficulty procuring their supplies, sometimes even leaving the operating room, thus, delaying the anesthesia preparation time. Again, a multidisciplinary approach is needed for this aspect through close communication with the pharmacy services. The supplies required by the doctors are made available to decrease waiting and preparation time.

The surgical preparation time is prolonged for the Plastics, Retina, and Orbit services since there were additional procedures such as examination under anesthesia (for retinal drawings), skin markings, local anesthesia, and nasal packing which were done during this time.

Across age groups, it was noted that anesthesia preparation times were significantly shorter for pediatric patients since they were induced first before the adult cases, with the two anesthesiologists rotating in the department assisting each other in the induction. Only after the induction of the pediatric case will the other anesthesiologist begin inducing the adult patient. Thus, the other room with the adult case would have a prolonged anesthesia preparation time. Furthermore, trans-out lag times were also significantly decreased in pediatric cases. An observed practice that may have reduced trans-out time was carrying the pediatric patients to the PACU, which was only thirty meters away, unlike adult patients wherein a bed was still needed for transfer.

Total turnaround time (TAT) is the period from the exit of the first patient until the start of the next patient's surgery. It is a total of three-time segments, namely, in chronological order, "exit lag," "turnover time," and "entry lag." Exit lag is the time from the end of surgery until the exit of the patient; turnover time is the time from the exit of the patient until the entry of the next patient, while entry lag is the time from entry of the following patient until the start of surgery. The TAT for both adult and pediatric cases was more than one hour for both groups. The analysis showed that for both age groups, "entry lag" (time from patient in until the start of surgery) had the most significant effect on total TAT. However, it must be noted that the limited number of information regarding turnaround time among both adult and pediatric patients undergoing surgery may render these regression equations to be lacking in precision. Nevertheless, this result can direct the hospital administration in introducing interventions in the segment that has the most significant effect on efficiency.

Benchmarking results of this study showed unsatisfactory results (below $50^{\text {th }}$ percentile), except for room turnover time, which was above the $95^{\text {th }}$ percentile. However, the exact setting of this study may not correspond to the centers included in the database. Likewise, there was no discrimination among types of surgery, subspecialty, or health center in their benchmarks, so results should be taken cautiously. Despite these reasons, the benchmarking results still give us an idea of the standard that the department can aim to improve its efficiency.

Lapitan et al. conducted a similar study at the Department of Surgery of the Philippine General Hospital. ${ }^{6}$ Similar results have been found in their study, with $3.9 \%$ of cases starting on time and $49.7 \%$ starting more than one hour later. Comparing results between studies showed shorter mean and median durations for anesthesia lag time and surgical lag time in the Department of Ophthalmology, while surgery preparation and wake up time were shorter in the Department of Surgery. ${ }^{6}$ It is notable that there was not much difference in induction time for both operating room complexes, possibly because both operating theaters use the same set of anesthesiologists.

\section{Limitations}

This study is limited in that the exact arrival times of all doctors were not noted. Likewise, there were only general observations of the processes as they occurred. The exact reasons for specific delays were not obtained since direct interaction and inquiry of the study observer with the department staff could affect measurements. Lastly, future researches to study more cases and the effect of interventions can be done.

\section{CONCLUSION}

This is the first study on operating room efficiency for ophthalmology surgeries under general anesthesia published in the country. Results showed that differences 
in surgical practices among subspecialties and age groups affected efficiency. Likewise, this research determined which particular parameters needed the most improvement and had the most effect on overall turnaround time. Lastly, this study highlights the need for a multidisciplinary approach in operating room efficiency, with all stakeholders agreeing to set standards and goals. Overall, results can serve as useful baseline data for future research and guide hospital administrators to determine improvements in efficiency and quality health care.

\section{Statement of Authorship}

MNU - Conceptualization and study design, data collection, analysis, writing and approval of final manuscript.

TRC - Conceptualization and study design, critical revisions to the paper, and approval of final manuscript.

\section{Author Disclosure}

Both authors declared no conflicts of interest.

\section{Funding Source}

This paper is funded by the Philippine General Hospital Expanded Health Research Office (PGH-EHRO) Residents' Research Grant 2018.

\section{REFERENCES}

1. Oh HHC, Phua TB, Tong SC, Lim JFY. Assessing the performance of operating rooms: What to measure and why? Proc Singapore Healthc. 2011; 20(2):105-9. doi:10.1177/201010581102000206

2. Childers CP, Maggard-Gibbons M. Understanding costs of care in the operating room. JAMA Surg. 2018 Apr; 153(4):e176233. doi:10.1001/jamasurg.2017.6233

3. Wong J, Khu KJ, Kaderali Z, Bernstein M. Delays in the operating room: Signs of an imperfect system. Can J Surg. 2010 Jun; 53(3): 189-95.

4. Foster T. Data for benchmarking your OR's performance. OR Manager. 2012; 28(1):13-6.

5. Glossary of times used for scheduling and monitoring of diagnostic and therapeutic procedures. AORN J. 1997 Oct; 66(4):601-6. doi:10.1016/s0001-2092(06)62913-x.

6. Lapitan MCM, Buckley BS, Abalajon DD, Cruz PLO, Raymundo MEM. Efficiency status of the elective non-cardiac surgery operating rooms of the Department of Surgery of the Philippine General Hospital. Acta Med Philipp. 2013; 47(4):30-5. 